Pflügers Arch. 358, 203-212 (1975)

(C) by Springer-Verlag 1975

\title{
Phase Shift in the REM Sleep Rhythm*
}

\author{
Hartmut Schulz, Gerhard Dirlich, and Jürgen Zulley \\ Max-Planck-Institut für Psychiatrie, München, Germany
}

Received April 10, 1975

\begin{abstract}
Summary. The periodic alternation between REM and NREM sleep was analyzed. Usually, sleep records of consecutive nights of a subject are regarded to be independent events. However, it may be that consecutive nights are realizations of a continuously ongoing rhythm. This was tested in the present study. The temporal patterns of REM and NREM sleep in sequences of about 30 consecutive nights for 3 subjects were analyzed. The results show that only the onset of the first REM sleep phase during any one night may be predicted from the sleep onset time, whereas a systematic phase shift between consecutive nights was observed in the later REM sleep phases. Thus, the onset of later REM sleep phases is better predicted by assuming a rhythm with stable period length which controls the appearance of REM sleep phases in successive nights. Under the experimental conditions the phase shift was between 5 and $10 \mathrm{~min}$ per $24 \mathrm{hrs}$ for the 3 subjects. The result is accordance with Kleitman's basic rest activity cycle (BRAC) hypothesis.
\end{abstract}

Key words: Sleep - Circadian Rhythm - Ultradian Rhythm.

The two most notable features in the appearance of REM sleep phases during the course of one night are the increase of the average length of the REM sleep phases (Webb, 1971) and the periodic alternation between REM and non-REM (NREM) sleep phases (Dement and Kleitman, 1957). In an appropriate model for the description of the REM sleep patterns against time, therefore at least two factors must be included, corresponding to these features. The work reported here deals with the factor which is responsible for the REM sleep rhythmicity. Three hypotheses for the phase control of the REM sleep rhythmicity are compared.

Analyses of the rhythmicity in sleep records are commonly carried out for single nights, the results then being averaged over several nights. This procedure requires a choice of time reference for all recorded nights. Proposed time references include local time (Globus, 1966) and the time of sleep onset (Lubin, 1974). The use of a particular time reference system implies the assumption of an entrainment of the REM sleep rhythm by synchronizers which are locked to the respective system. Using the above mentioned time references, a phase reset according to local time or to sleep onset time respectively is assumed. Consecutive

* This work was supported by the Deutsche Forschungsgemeinschaft (Le $193-9 / 10)$. 
sleep records are treated as independent observations in a repeated experiment.

A different approach is based on Kleitman's hypothesis of the basic rest activity cycle (BRAC) (Kleitman, 1969). In this case the REM sleep rhythm is taken to be the result of a periodic process, which is not limited to sleep, but continues throughout wakefulness. According to the BRAC hypothesis a periodic factor which is continuously present in the long term, possibly over several successive sleep records, is the synchronizer of the REM sleep rhythm. Under this assumption consecutive sleep records have to be treated as discrete observations of a unique process. Therefore, the time reference used in an averaging procedure has to be chosen in such a way that it links together points of equal phase in the triggering BRAC process during the recorded nights. A special case occurs when the period of the BRAC or REM sleep rhythm is equal to a submultiple of $24 \mathrm{hrs}$, so that points of equal phase would appear to be synchronized with local time. In all other cases the points of equal phase of the REM sleep rhythm occur with constant time shift in consecutive nights. Any analysis must allow for this feature by the choice of a time reference system with a constant time shift between successive nights.

The three models for synchronization of REM sleep rhythm, as described above, are investigated in the following.

\section{Method}

Subjects. The sleep data of 3 subjects were analyzed. Two subjects (BI and KI) were male, 24 and 31 years old, the third subject (SI) was female, 20 years old. All were healthy unmarried students without sleep problems. They received payment for sleeping in the laboratory.

Design and Procedure. The subjects slept for 29, 30 and 31 undisturbed consecutive nights in the laboratory (see Table 1).

They came to the laboratory about $1 \mathrm{hr}$ before going to bed. Questionnaires were filled out and electrodes for EEG, EOG and EMG monitoring were fixed. Lights were turned off between 10:30 and 12:00 p.m.

Table 1. Characteristics of subjects, number of nights and "lights off" time

\begin{tabular}{|c|c|c|c|c|c|}
\hline \multirow[t]{2}{*}{$S \mathrm{~s}$} & \multirow[t]{2}{*}{ Sex } & \multirow[t]{2}{*}{ Age } & \multirow{2}{*}{$\begin{array}{l}\text { Number of } \\
\text { nights }\end{array}$} & \multicolumn{2}{|c|}{ "Lights off" (local time) } \\
\hline & & & & $M$ & $s(\min )$ \\
\hline BI & male & 24 & 30 & $23^{20}$ & \pm 15 \\
\hline KI & male & 31 & 31 & $23^{33}$ & \pm 37 \\
\hline SI & female & 20 & 29 & $23^{30}$ & \pm 17 \\
\hline
\end{tabular}

$M$ mean.

$s$ standard deviation. 
The subjects slept for 7 to $8 \mathrm{hrs}$ in a sound-attenuated, electrically shielded room. The adjacent recording room was equipped with an 8- or 12-channel Mingograf and an Ampex PR 500 tape recorder. At least 2 unipolar vertex EEGs (C3-A2, C4-A1), 2 unipolar, horizontal EOGs, chin EMG, EKG and an actogram from the bedsprings were recorded.

Scoring. All sleep records were scored visually by one or two scorers in $30 \mathrm{sec}$ time epochs according to the criteria of Rechtschaffen and Kales (1968). For the present investigation only REM sleep was distinguished from the other sleep stages 1, 2, 3 and 4. Thus each 30 sec epoch was either scored REM or NREM. Any NREM activity shorter than 5 min which appeared during REM sleep was also scored REM. The recorded data were therefore reduced to blocks of REM and NREM epochs.

Computational Procedures. The following procedures were used to obtain data for an intra-individual comparison of the three suggested models for synchronization of the REM sleep rhythm .

The proposed models are (1) synchronization of the REM sleep rhythm to local time, (2) synchronization to a continuing BRAC process, and (3) synchronization to the respective time of sleep onset for each night. For each model, all sleep records were referred to the appropriate time reference system. The REM sleep frequency at time $t$ is defined as the number of nights during which REM sleep was scored at time $t$, divided by the total number of nights. This function is smoothed using a moving average over $45 \mathrm{~min}$.

For model (1) where the time coordinate system is local time, the corresponding REM sleep frequency function is denoted as $r_{1}(t)$ in the following.

With model (2) the period of the driving BRAC process is unknown, and therefore the expected time shift for successive nights between points of equal phase must be estimated from the observed data. For this purpose a time coordinate system was used in which successive sleep records were shifted by a constant amount against local time. This time shift was used as the parameter to generate a set of frequency functions. The one function which best displays the periodicity of the REM sleep phases could than be determined from this set of functions. The time shift from the selected function was taken as the estimate for the actual time shift between points of equal phase in the REM sleep process. This REM sleep frequency function is denoted as $r_{2}(t)$,

In model (3) each sleep record was shifted against local time according to time of sleep onset, so that all sleep onset times were positioned at zero. The REM sleep frequency function $r_{3}(t)$ was calculated using this time coordinate system.

\section{Results}

The results of the data analysis for subject BI are shown in Figs. 1-3.

In Fig. 1 the REM sleep data are presented according to the local time model. Fig. 1a shows the temporal pattern of the REM sleep phases in each night. The $x$-axis represents time in increments of 30 sec. The 30 nights are displayed in their natural order. REM phases are indicated by solid lines, NREM phases are left blank. The dot at the left end of each pattern marks the sleep onset time-which is defined as the first 30 sec epoch of sleep stage 1. 


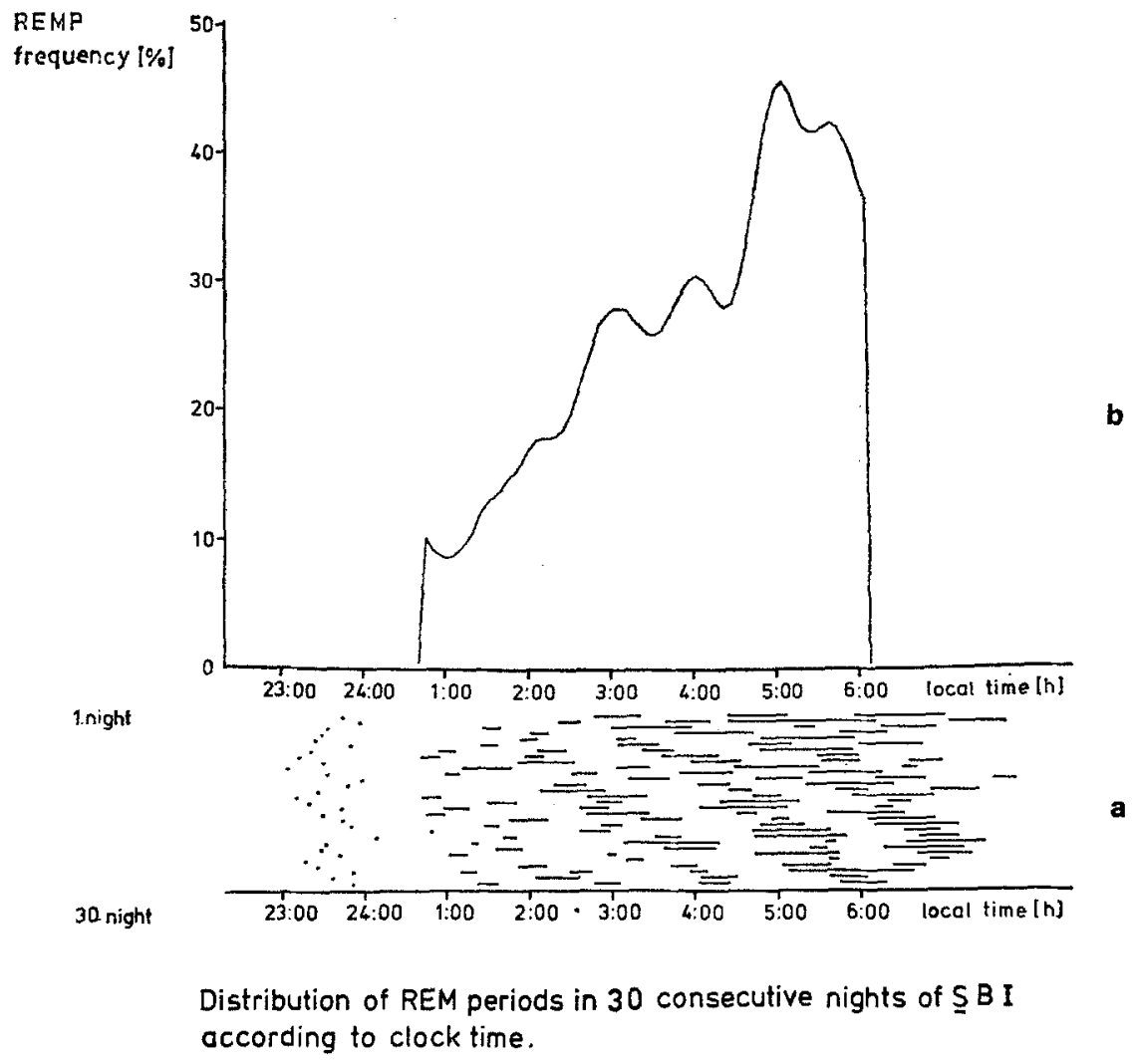

Fig. $1 \mathrm{a}$ and $\mathrm{b}$. Distribution of REM sleep periods in 30 consecutive nights of subject BI according to local time. (a) Arrangement of the 30 nights in their natural order from top to bottom. REM sleep periods are black, NREM sleep periods are left blank. The dot at the left end of each night marks the first sleep stage 1 epoeh

(sleep onset). (b) Smoothed REM sleep frequency function $\mathbf{r}_{\mathbf{1}}(t)$

Fig. $1 \mathrm{~b}$ contains the REM sleep frequency function $r_{1}(t)$. The increase in REM sleep frequency with increasing sleeping time is obvious. However, a certain amount of information in the raw data in Fig. 1 a is lost due to this summation procedure. In Fig. 1 a a pattern of oblique stripes can be recognized in which the REM frequency appears to oscillate. This feature of the data is lost in Fig. $1 \mathrm{~b}$.

Fig. 2 shows the same data set. Here, the data are related to a time coordinate system with a time shift of +5.0 min between consecutive nights [model (2)]. The resulting smoothed frequency function is strikingly periodical. The time shift of $+5.0 \mathrm{~min}$ between consecutive nights 


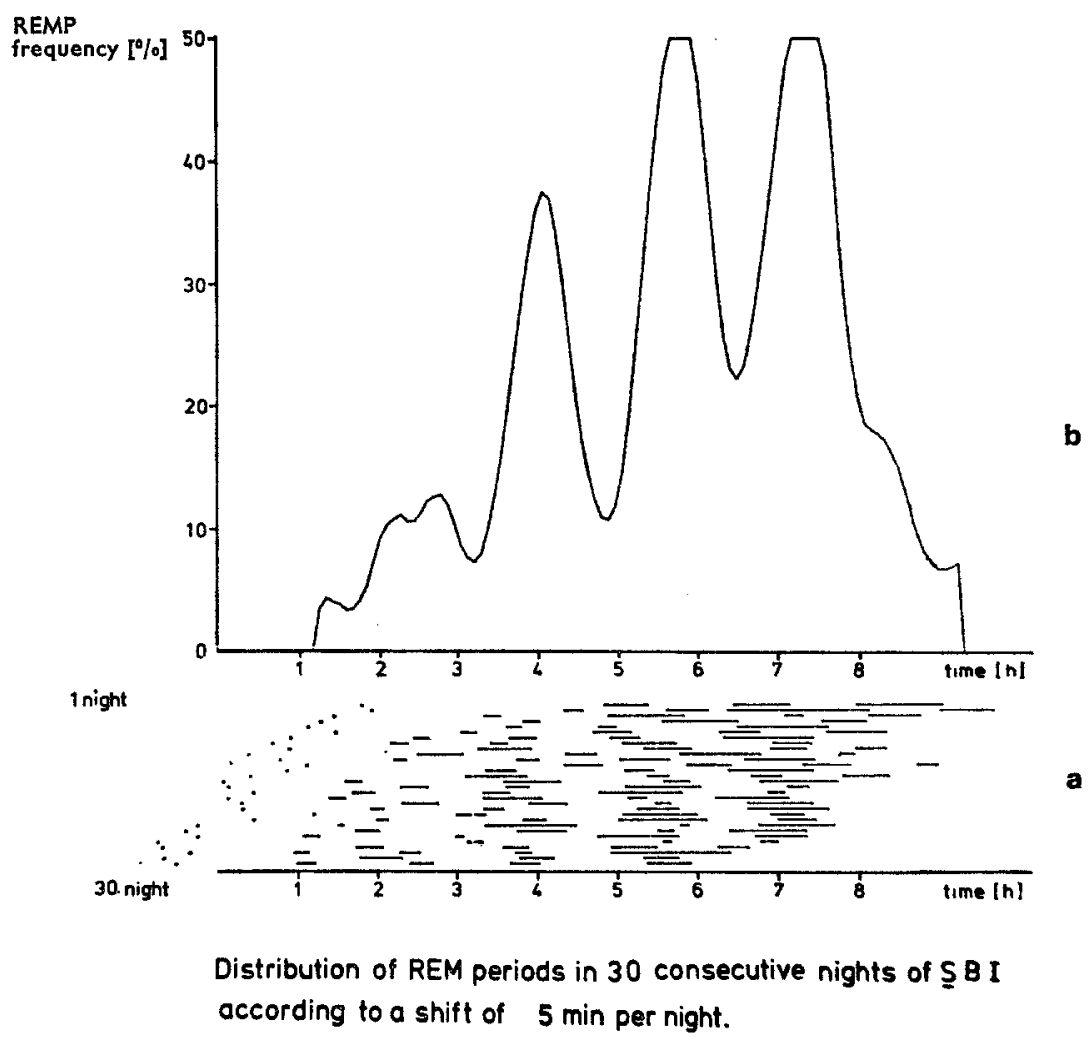

Fig. $2 a$ and $b$. Distribution of REM sleep periods in 30 consecutive nights of subject BI, with a time shift of 5.0 min per night. (a) Arrangement of the nights in their natural order from top to bottom. (b) Smoothed REM sleep frequency function $r_{2}(t)$

turned out to yield the best representation of the periodicity within a set of shift values ranging from -10 to +14 min with a stepwidth of $0.5 \mathrm{~min}$. day.

The estimated phase shift for this subject is therefore 5.0 min per

In Fig. 3 the same data are arranged taking sleep onset in each night as the common reference point [model (3)]. The resulting REM frequency function $r_{3}(t)$ shows both the overall REM sleep frequency increase and the refractory interval of about $70 \mathrm{~min}$ after sleep onset; this is followed by a steep increase due to the first REM sleep phase.

A comparison of Figs. 2 and 3 leads to the conclusion that onset of the first REM sleep phase is correlated with sleep onset, while the 


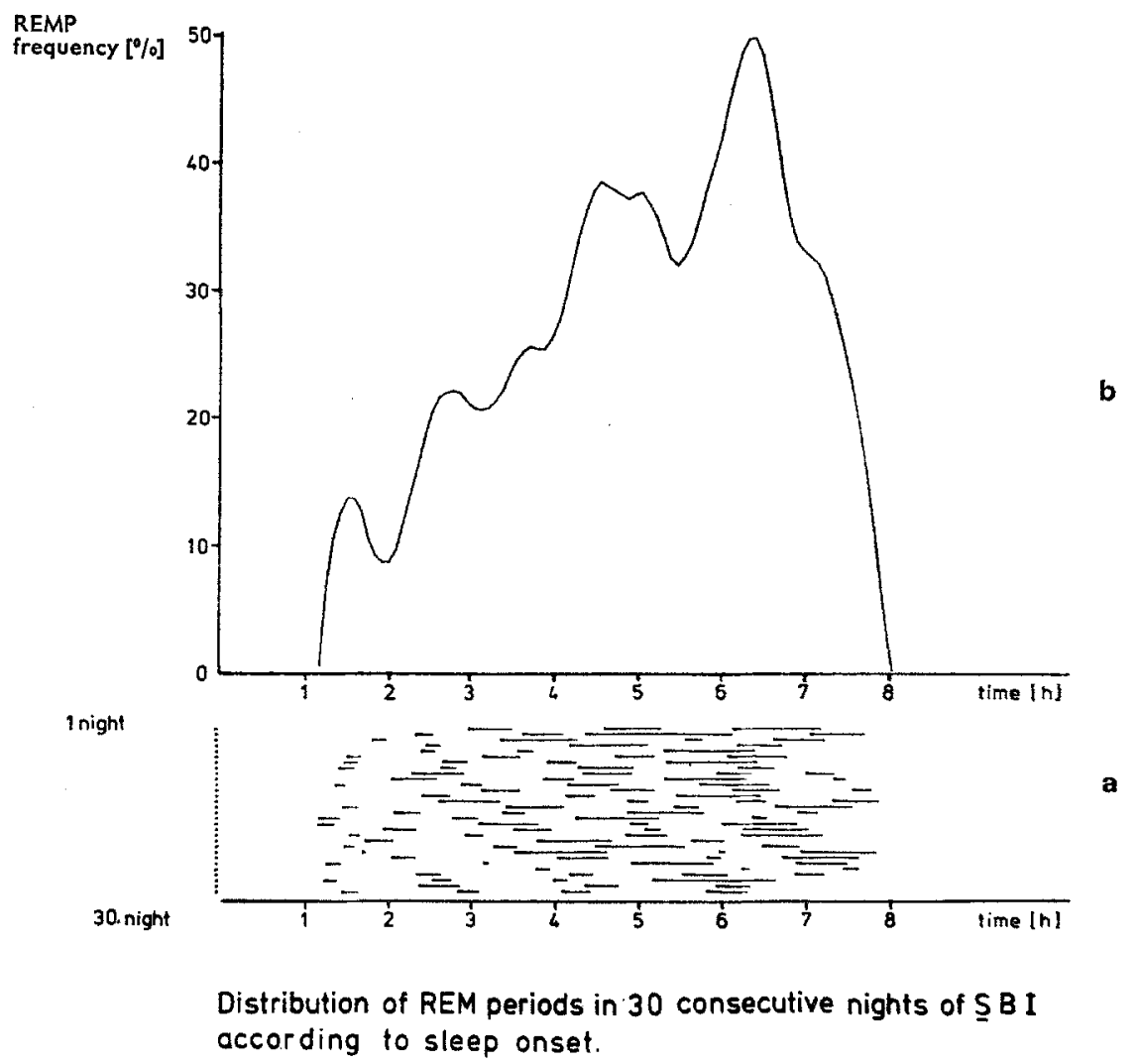

Fig. 3 a and b. Distribution of REM sleep periods in 30 consecutive nights of subject $\mathrm{BI}$ according to sleep onset. (a) Arrangement of the nights in their natural order from top to bottom. (b) Smoothed REM sleep frequency function $r_{3}(t)$

appearance of later REM sleep phases is better described by a model with constant phase shift between consecutive nights.

The data sets of the other subjects were processed in the same way. With a variable time shift according to the above stated procedure 50 REM sleep frequency functions were computed for each subject. A meaningful comparison of these functions was restricted, on amount of the time shift, to that time in which the records of all nights overlapped. This amounted to a length of $250 \mathrm{~min}$.

Fig. 4 shows two measures resulting from this comparison. The plots from left to right correspond to subjects BI, SI and KI respectively. In Fig. $4 \mathrm{a}$ the measure $\mathrm{A}(s)$ of the periodic component in each REM frequency function is plotted against the time shift in increments of $0.5 \mathrm{~min} . \mathrm{A}(s)$ is the minimum amplitude difference between adjacent 

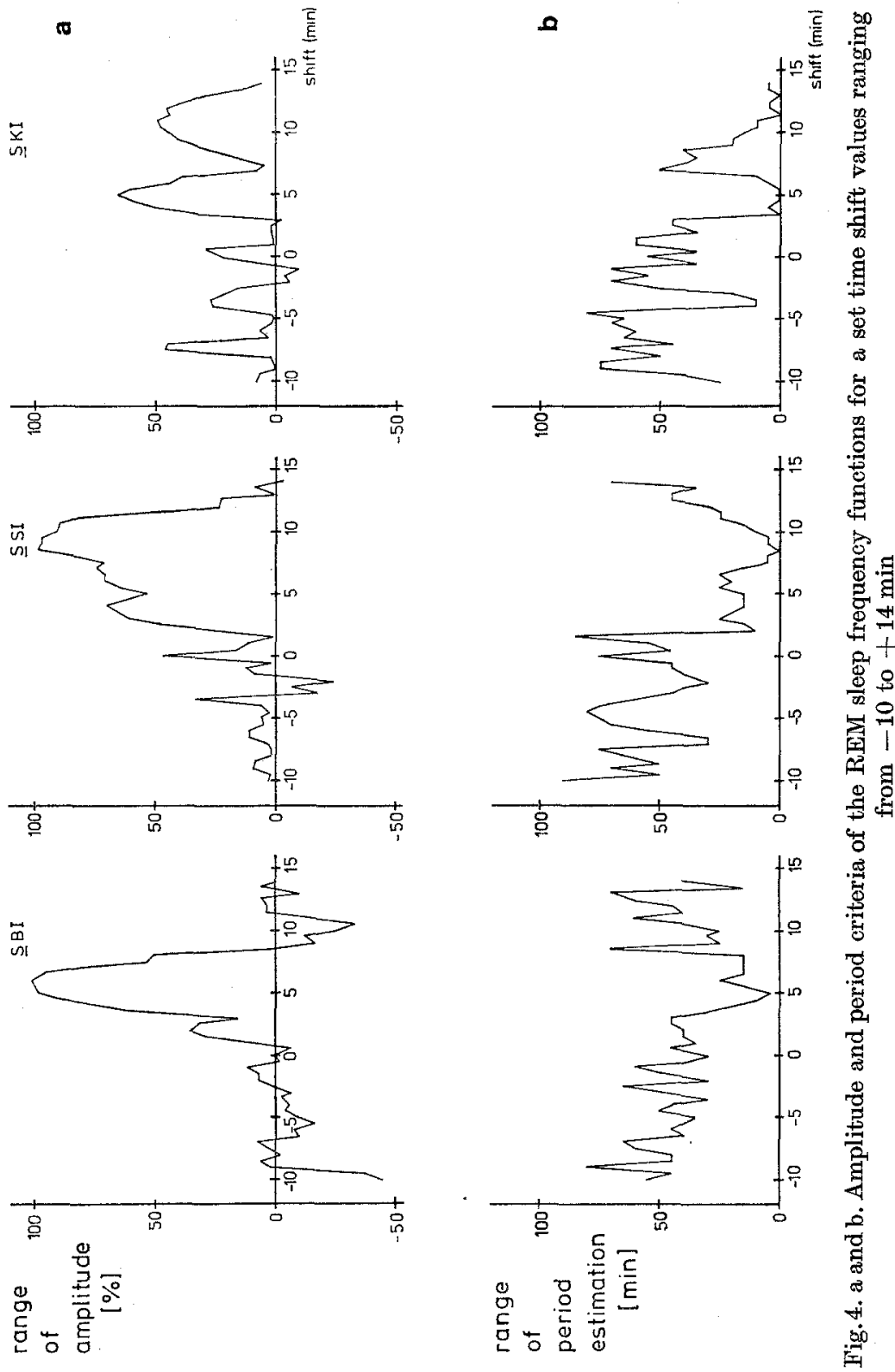

extreme values for each REM frequency function obtained for the different time shifts $s$. The amplitudes are expressed in percent of the mean amplitude of the REM frequency function. 
In Fig. $4 \mathrm{~b}$ a measure $\mathrm{P}(s)$ of the period stability of the periodic component in the REM frequency function is plotted against time shifts $s$. The measure $\mathrm{P}(s)$, with a resolution of 5 min units, is the variability in the set of period estimates obtained from the peak-to-peak and trough-to-trough measures within the $250 \mathrm{~min}$ interval.

A comparison of these measures for the three subjects yields the following findings. For subject BI the best representation of the period is obtained for shift values of about $5 \mathrm{~min}$ per day. At $s=5.0 \mathrm{~min}$ per day $\mathrm{A}(s)=99 \%$, and $\mathrm{P}(s)=5 \mathrm{~min}$. For subject $\mathrm{SI}$ the best description of the periodic is obtained for a shift of about $8 \mathrm{~min}$ per day. At $s=8.5 \mathrm{~min} \mathrm{~A}(s)=98 \%$ and $\mathrm{P}(s)=0 \mathrm{~min}$. From the results for subject $\mathrm{KI}$, the measure $\mathrm{A}$ has no single distinct maximum, however for a time shift of $5 \mathrm{~min}$ per day the period estimate is constant and reaches a local maximum, at $s=5.0 \mathrm{~min}$ with $\mathrm{A}(s)=65 \%$ and $\mathrm{P}(s)=$ $0 \mathrm{~min}$.

\section{Discussion}

The main result of this investigation is the evidence for a drift of the REM sleep rhythm in successive sleep records. This observation supports the assumption of a synchronization of the REM sleep rhythm to an ultradian oscillation. This is consistent with Kleitman's BRAC hypothesis, according to which a continuous periodic process operates throughout sleep and wakefulness. The constant phase shift of the REM sleep rhythm, observed in sleep recordings of 30 consecutive nights, suggests that the $\mathrm{BRAC}$ period is stable. Under the reported experimental conditions for all 3 subjects, phase shifts in the range of 5 to $10 \mathrm{~min}$ per $24 \mathrm{hrs}$ were found. Comparable phase shifts in the appearance of penile erections in sleep of different nights were reported in this journal almost 30 years ago by Ohlmeyer and Brilmayer (1947). They reported that the mean duration of the erection cycle during sleep (Ohlmeyer et al., 1944) was not an integral submultiple of $24 \mathrm{hrs}$ and consequently they assumed a phase shift of the underlying process against local time.

Until now the BRAC hypothesis for the adult has been supported by similarities between period estimates from data in sleep experiments (REM sleep rhythm) and period estimates of other ultradian rhythms from data of awake subjects, e.g. oral activity (Friedman and Fisher, 1967; Oswald et al., 1970), EEG measures (Sterman et al., 1972; Stevens et al., 1971), and special patterns of EOG activity (Othmer et al., 1969). The reported investigation presents another approach to test the BRAC hypothesis. The observed systematic drift of the REM sleep rhythm may be explained by the assumption of a persistent driving process with stable period. 
Model (1), which assumes a phase coupling of the REM sleep rhythm with local time, is not supported by the reported experiment. An optimal 0-min time shift of the REM sleep rhythm was not observed in the 3 subjects.

The results of the experiment support the assumption that the two factors which control the appearance of REM sleep phases, i.e. sleep onset [model (3)] and BRAC [model (2)], are restricted to different times during each night. This result is in accord with observations on the organization of REM sleep in monkeys (Kripke, 1973, pp. 317-318). In all sleep records sleep onset is followed by a $40-70$ min time interval without REM sleep and the onset of the first REM sleep phase is still determined by the time of sleep onset. Thus REM sleep control by BRAC obviously requires that the subject be freed from the influences of external time givers for some time. This condition holds after the subject has passed the first sleep cycle.

We thank Mrs. Mechthild Bär for scoring of the sleep records, Dr. Laverne Johnson and coworkers for critical reading of a first version of this paper and Mr. Andy Clarke for checking the English version of the paper.

\section{References}

Dement, W., Kleitman, N.: Cyclic variations in EEG during sleep and their relation to eye movements, body motility, and dreaming. Electroenceph. clin. Neurophysiol. 9, 673-690(1957)

Friedman, S., Fisher, C.: On the presence of a rhythmic, diurnal, oral instinctual drive cycle in man: A preliminary report. J. Amer. psychoanal. Ass. 225, $959-960$ (1967)

Globus, G. G.: Rapid eye movement cycle in real time. Arch. gen. Psychiat. 15, $654-659$ (1966)

Globus, G. G.: Quantification of the sleep cycle as a rhythm. Psychophysiology 7, $244-253(1970)$

Kleitman, N.: Basic rest activity cycle in relation to sleep and wakefulness. In: Sleep physiology and pathology, A. Kales (Ed.), pp. 33-38. Philadelphia: Lippincott 1969

Kripke, D. F.: Ultradian rhythms in sleep and wakefulness. In: Advances in sleep research, Vol. I, E. D. Weitzman (Ed.), pp. 305-325. Flushing, New York: Spectrum Publications 1974

Lubin, A.: Personal communication. (Letter from 4. Sept. 1974)

Ohlmeyer, P., Brilmayer, H.: Periodische Vorgänge im Schlaf. Pflügers Arch. ges. Physiol. 249, 50-55 (1947)

Ohlmeyer, P., Brilmayer, H., Hüllstrung, H.: Periodische Vorgänge im Schlaf. Pflügers Arch. ges. Physiol. 248, 559-560 (1944)

Oswald, I., Merrington, J., Lewis, H.: Cyclical "on demand" oral intake by adults. Nature (Lond.) 225, 959-960 (1970)

Othmer, E., Hayden, M. P., Segelbaum, R.: Encephalic cycles during sleep and wakefulness in humans: $A$ 24-hour pattern. Science 164, 447-449 (1969) 
Rechtschaffen, A., Kales, A.: Manual of standardized terminology. Techniques, and scoring system for sleep stages of human subjects. National Institutes of Health Publication No. 204, Washington. Superintendent of Documents. Book $1-62(1968)$

Sterman, M. B.: The basic rest-activity cycle and sleep: Developmental considerations in man and cats. In: Sleep and the maturing nervous system.C.D.Clemente, D. P. Purpura, and F. E. Mayer (Eds.), pp. 175-197. New York: Academic Press 1972

Stevens, J. R., Kodama, H., Lonsbury, B., Mills, L.: Ultradian characteristics of spontaneous seizure discharges recorded by radio telemetry in man. Electroenceph. clin. Neurophysiol. 31, 313-325 (1971)

Webb, W. B.: Sleep behavior as a biorhythm. In: Biological rhythms and human performance, W. P. Colquehoun (Ed.), pp. 149-178. New York: Academic Press 1971

\author{
Dr. Hartmut Schulz \\ Max-Planck-Institut \\ für Psychiatrie \\ D-8000 München 40 \\ Kraepelinstraße 10 \\ Federal Republic of Germany
}

